\title{
Editorial
}

\section{Open science, a complex movement}

\begin{abstract}
Science must be open and accessible, and diffusion of knowledge should not be limited by patents and copyrights. After the Open Science Summit held in Berkeley, some notes about sharing scientific data and updating the social contract for science. Against the determinist view on technological and legal solutions, we need an explicit reflection on the relation between science and society. Both academic and industrial science seem unable to fulfill open science needs: new societal configurations are emerging and we should keep asking questions about appropriation, power, privatisation and freedom.
\end{abstract}

Science must be open and accessible, and diffusion of knowledge should not be limited by patents and copyrights. After the wave of legal, political and social clashes that shook science because of the rise of intellectual property rights for scientific knowledge and data, now we need to "update the social contract for science." This was the subtitle of the first edition of the Open Science Summit (OSS), ${ }^{1}$ a conference held at the University of California in Berkeley from July 29th to 31st. The OSS gathered scientists, researchers, social entrepreneurs, non-profits and science policy experts to discuss the strategies and possible evolutions of the open science movement.

But how do we define Open Science? According to Jason Hoyt of Mendeley, ${ }^{2}$ science is open when "it is available to any one in the world to do whatever they like with it." Yale's Victoria Stodden tried to refine this position: "open code is as much an important part of this as much as open data". Stodden's proposal is related to reproducibility: a publishing standard which includes analytical tools, raw data and experimental protocols, giving any scientist the possibility of reproducing a colleague's experiment. But, as Stodden puts it, "we are not updating the social contract, what we're doing is returning to the scientific method which has been around for hundreds of years. It is what a scientist is supposed to do." Yet there is a cultural problem: "our adaptation to the technological tools for openness and sharing is not happening fast enough, and is bringing about a credibility crisis" of science. And there is also a generational problem: "younger scientists want to share everything. Older leaders must not just give examples, but also try to provide tools for them to be open." This means that we need to forge new legal and societal tools for open science. But we also need to protect young researchers from the "existential crisis" they experience when they switch from a world in which everything is shared, i.e., social networks, to one in which knowledge is private. But can we just rely on technological solutions and on a revival of $20^{\text {th }}$ century open science culture?

Michael Neilsen, researcher and blogger, argued that we need "to create new ways for scientists to create reputation, based on new tools," since the incentive system that drives the scientist's work (to publish in peer reviewed journals) prevent the community from adopting new solutions. "Today's subsidies prevent science from adopting new technologies and new solutions - scientists are motivated to reveal discoveries in older media. Therefore the first open science revolution - the sharing of results in journals - is now hindering the second open science revolution." Now we need a new system which distributes benefits to those scientists who decide to openly share their knowledge and data. This approach seems to be more attuned to the current needs of open science: along science history, its communication and publishing systems have always developed in order to respond to the incentives society gives them.

What could an updated social contract look like, then? In Berkeley there was no room for an explicit reflection on the relation between science and society and the role of communication technologies and practices. Nevertheless, judging from the talks at OSS, in the future companies will have an even bigger role in scientific research. Open sharing of knowledge and data could become just another business model for corporate R\&D. According to Stanford's Drew Endy, "in 15 to 30 years something really interesting will develop between these two poles: FLOSS (Free, libre and open source software) and synthetic biology." Both companies and individuals will be able to make key innovations outside the 
walls of universities. And in Berkeley there were a lot of small companies, foundations, non-profits, social entrepreneurs and start ups which use open source models of innovation.

We don't know if this emerging movement will succeed in providing crucial scientific innovation. But there is at least one thing those actors are providing: a new, broader meaning of "open science", which is not only the free circulation and sharing of information within the scientific community. An important part of the open science movement is more and more represented by people who innovate and conduct research outside the boundaries of scientific institutions. Their radical claims for openness and access to scientific knowledge is heating up a debate on the boundaries of contemporary science: on one side, citizens participation in its decisional processes; on the other, in the scientific enterprise itself. A few examples presented in Berkeley include DIYbio, ${ }^{3}$ the network of garage biologists from which projects such as OpenPCR ${ }^{4}$ and the community lab Biocurious ${ }^{5}$ are stemming; MyDaughtersDna, ${ }_{6}^{6}$ an open platform for the sharing of information about genetic pathologies to researchers, physicians and patients; the Pink Army Cooperative, ${ }^{7}$ a non-profit co-op with an open source attitude which works on personalized medicine for cancer - "the first DIY pharmaceutical company."

New forms of funding research - or, if you prefer, new ways of appropriating its results - are fueling open science, and the old scientists' culture is not enough to explain the new configuration of science and society interactions. The picture drawn during the OSS is a vital and dynamic movement from a societal and technological point of view. At the same time it is a complex and diverse movement. Academic and industrial science seem unable to fulfill its needs. There are new and emerging actors playing a growing role in its development, and also new societal configurations and new technological tools. If we want to avoid determinism, we can not just advocate for the widespread adoption of open platforms and licenses and for a return to $20^{\text {th }}$ century open science culture. We need to dig into the science and society relationship and keep on asking questions about appropriation, power, privatization and freedom.

Alessandro Delfanti

\section{Notes and references}

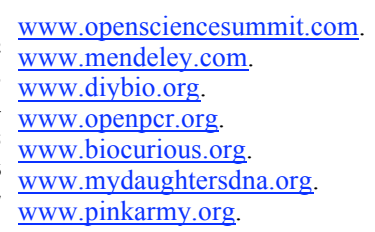

How TO CITE: $\quad$ A. Delfanti, Open science, a complex movement, Jcom 09(03) (2010) E. 